\title{
Métodos de estimación de la exactitud posicional a través de elementos lineales
}

\author{
Izar Sinde González
}

Recibido el 12 de mayo de 2014; aceptado el 14 de julio de 2014

\begin{abstract}
Currently exists several developed and in process of development methods that aim to check the positional accuracy in cartography. The most extended are based in punctual features (NMAS EMAS ASPRS or NSSDA) but exists methods based on lineal elements and even on surface elements. As a result of some recent researches, this article details the most extended methods to check the positional accuracy with lineal elements. It also indicates what would be future investigation guidelines to follow on this matter. For this task, some application cases in several cartographic specialties such as coastal cartography, cadaster, roads or simply the error made by applying various generalization processes to lineal features will be analyzed. With exception of the latter, the other specialties require to get extra data from a more reliable source. Data gathering process is also analyzed because it is the greatest constraint for the application of the aforementioned methods. The advancement of new technologies as well as the geographic databases characteristics, in which usually are stored lineal features, make these methods a way to check the positional accuracy to consider in the future and that will be able to compete whit the already standardized punctual element based methods.
\end{abstract}

Key words: lineal feature, positional accuracy, cartography, quality, geometry.

\section{Resumo}

Atualmente existem diferentes métodos desenvolvidos e em processo de pesquisa que têm como objetivo comprovar a exatitude posicional na cartografia. Os mais comuns e padronizados são os baseados em elementos pontuais (NMAS EMAS ASPRS ou NSSDA) mas, também existem os baseados em elementos lineares in-

* Escuela Técnica Superior de Ingenieros en Topografía, Geodesia y Cartografía, Universidad Politécnica de Madrid (UPM), Av. Tierra Llana 86-88 $6^{\circ}$ D, Villalba, Lugo, correo electrónico: i.sinde@alumnos.upm.es 
cluindo-se os superficiais. No presente artigo se detalha quais são os métodos mais comuns de comprovação da exatitude posicional com elementos lineares, que resultados se obteve em alguns estudos recentes e quais serão as futures linhas de investigação a se seguir nesta temática. Para esta tarefa, se analisou casos de aplicação em diversas especialidades da cartografia tais como cartografia costeira, cadastro, vias de transporte ou simplesmente o erro ao se aplicar algum processo de generalização a elementos lineares. Exceto na generalização, as demais variáveis necessitam da obtenção de dados adicionais de alguma fonte mais confiável. A forma que se pode adquirir estes dados também será analisada neste documento, já que é aí que se encontra o maior condicionante para a aplicação das citadas metodologias. O avanço das novas tecnologias assim como as características das bases de dados geográficos, nas quais predomina em geral os elementos lineares, fazem destes métodos uma forma de comprovação da exatitude posicional a se ter muito em conta e que se poderia competir com as padronizações baseadas em elementos pontuais.

Palavras chave: elemento linear, exatitude posicional, cartografia, qualidade, geometría.

\section{Resumen}

En la actualidad existen diferentes métodos desarrollados y en proceso de investigación que tienen como objetivo comprobar la exactitud posicional de la cartografía. Los más extendidos y estandarizados son los basados en elementos puntuales (NMAS EMAS ASPRS o NSSDA), pero también existen los basados en elementos lineales e incluso superficiales. En el presente artículo se detallan cuáles son los métodos más extendidos de comprobación de la exactitud posicional con elementos lineales, qué resultados se han obtenido en algunos estudios recientes y cuáles serán las futuras líneas de investigación a seguir en esta temática. Para esta tarea, se analizan casos de aplicación en diversas especialidades de la cartografía como pueden ser la cartografía costera, el catastro, vías de comunicación o simplemente el error al aplicar algún proceso de generalización a elementos lineales. Excepto la generalización, las demás variantes requieren obtener datos adicionales de alguna fuente más fiable. La forma en que se pueden adquirir estos datos se analizará también en este documento, ya que es aquí donde radica el mayor condicionante para la aplicación de las citadas metodologías. El avance de las nuevas tecnologías así como las características de las bases de datos geográficas, en las cuales predominan en general los elementos lineales, hacen de estos métodos una forma de comprobación de la exactitud posicional a tener muy en cuenta y que podría competir con las ya estandarizadas basadas en elementos puntuales.

Palabras clave: elemento lineal, exactitud posicional, cartografía, calidad, geometría. 


\section{Introducción}

Ruíz et al. (2009) afirman que durante los últimos años se han desarrollado numerosos métodos para comprobar cualquiera de las componentes que afectan a la calidad de la cartografía, ya sea a su exactitud, completitud, coherencia, exactitud temporal o a sus metadatos. Aquí se analizarán los métodos que evalúen la exactitud posicional $\mathrm{y}$, en concreto, aquellos que lo hacen apoyándose en elementos lineales.

Del mismo modo también señala que la exactitud posicional se puede comprobar mediante los mencionados elementos lineales, pero esto no es lo más habitual. Las técnicas más ampliamente utilizadas y aceptadas son las basadas en elementos puntuales, las cuales serán mencionadas en el siguiente apartado.

Además de estos últimos, también existen los basados en elementos superficiales, que si bien se han utilizado en algunos trabajos como Fernández-Castro (2008), no existe una bibliografía demasiado extensa sobre el tema.

Mozas (2007) menciona en su trabajo que la aplicación de estos métodos de comprobación de exactitud posicional tienen hoy sentido gracias a los avances tanto informáticos en general como de las técnicas para obtener elementos lineales de referencia necesarios de forma rápida y sencilla (mediante tecnología GNSS), cosa que hace unos años era impensable. Del mismo señala como una ventaja de estos métodos que la fuente de datos formada por los elementos lineales es mayoritaria en las bases cartográficas de hoy en día.

\section{Métodos de comprobación de la exactitud posicional mediante elementos puntuales}

Existen numerosas pruebas o test de comprobación de la exactitud posicional de cartografía a través de elementos puntuales. Muchos de ellos incluso instaurados y reconocidos por importantes asociaciones cartográficas. Se citan a continuación algunos de los más extendidos.

Test NMAS (National Map Accuracy Standards) del United States Bureau of the Budget utilizado para comprobar la calidad de la cartografía del United States Geological Survey (USGS) desde 1947, sirviendo de modelo para muchas instituciones cartográficas de todo el mundo.

Test EMAS/ASPRS (Engineering Map Accuracy Standard) / (American Society of Photogrammetry and Remote Sensing) desarrollado por la American Society of Civil Engineers (ASCI), el American Congress on Surveying and Mapping (ACSM) y la American Society of Photogrammetry and Remote Sensing (ASPRS), utilizado para especificar la exactitud de los mapas topográficos a gran escala en los Estados Unidos. 
Test NSSDA (National Standard for Spatial Data Accuracy) del FGDC (1998), por el cual las agencias federales norteamericanas que realicen labores de producción de datos cartográficos deben atenerse a los procedimientos impuestos por este estándar.

Todas las pruebas mencionadas están establecidas a nivel estándar de voluntario cumplimiento por alguna asociación, pero como sostiene Fernández-Castro (2008) el test NMAS es el clásico por excelencia. Este test es el más simple y de fácil interpretación por el usuario. Por otro lado, NMAS presenta algunos inconvenientes como la falta de indicación de una medida de la inexactitud presente en el mapa o la elección del procedimiento de muestreo. En cuanto al test NSSDA es la prueba de referencia en todos los organismos de la administración pública norteamericana relacionados con esta temática. Su principal ventaja radica en que a diferencia de los demás, no indica si el mapa es aceptado o denegado, si no que ofrece un índice de calidad de la cartografía en unidades reales sobre el terreno. En este caso, es el usuario el que establece los límites de aceptación en función de sus necesidades. Por último, la principal ventaja del test EMAS/ASPRS es que las diferencias entre las localizaciones de los puntos sobre el mapa y sobre la fuente de referencia están expresadas en términos de distancias sobre el terreno. Además, el límite de error aceptable puede ser definido por el usuario como en el NSSDA. Sin embargo, en el test NMAS la unidad de medida es la distancia sobre el mapa y los límites son fijados por el estándar.

\section{Métodos de comprobación de la exactitud posicional mediante elementos lineales}

Según señalan Mozas y Ariza (2008), aunque no tan reconocidos como los test basados en elementos puntuales, sí existen una serie de métodos por los cuales se puede comprobar la exactitud posicional de la cartografía a través de elementos lineales. Prácticamente la totalidad de ellos se encuentran en fase de investigación y ninguno de ellos ha sido recogido a nivel de un estándar de cumplimiento obligatorio o voluntario, pero sí se han realizado estudios teóricos que analizan el comportamiento de éstos.

Los métodos de este tipo más citados en la bibliografía son el de Distancias de Hausdorff de Abbas et al. (1995), el de la Banda Épsilon de Skidmore y Turner (1992), el de Orlados Simples de Goodchild y Hunter (1997), el de Doble Orlado de Tveite y Langaas (1999) y el de correspondencia de puntos de Kagawa et al. 1999).

Como se puede observar, todos estos métodos han sido ideados a finales del siglo pasado, pero la aplicación de los mismos se han realizado en trabajos bastante más recientes que se mencionarán en apartados posteriores de este artículo. 


\section{Métodos de comprobación de la exactitud posicional mediante elementos superficiales}

No existe en la bibliografía consultada ningún documento cuyo tema central sea la estimación de la exactitud posicional en cartografía mediante elementos superficiales, pero sí existen algunas soluciones como la aportada en Fernández-Castro (2008), la cual utiliza un método basado en superficies para comprobar la exactitud de los objetos geográficos del catastro español. Lo que se hace en ese trabajo es considerar como elementos superficiales de mayor exactitud una serie de cubiertas de edificios medidas en campo mediante métodos GNSS. Posteriormente, se eligen una serie de muestras con entidad superficial de la cartografía catastral de la zona y se comparan las superficies. En el caso de este trabajo se obtuvo que las cubiertas en el catastro estaban $4.66 \%$ sobreestimadas en un intervalo de confianza de (1.45\%- 7.88\%) para un nivel de significación de 95 por ciento.

A pesar de que el autor no relacionó los resultados del test descrito anteriormente con los que obtuvo mediante las pruebas basadas en elementos puntuales, concluyó que aunque los errores en la estimación de las superficies son asumibles al no superar el 5\% sí sería motivo de revisión al pensar en un catastro multipro-pósito.

\section{Fundamentos teóricos y procedimientos de los métodos de comprobación de exactitud posicional de elementos lineales}

\section{Distancia de Hausdorff}

Este método se basa en los trabajos de Abbas et al. (1995) y permite evaluar la exactitud planimétrica a partir de las distancias de Hausdorff de pares de objetos. El método propuesto utiliza la distancia de Hausdorff como herramienta matemática entre una pareja de líneas. El procedimiento consiste en:

I. Seleccionar una muestra de líneas en la base de datos $(X)$.

II. Seleccionar las mismas líneas en otra fuente de mayor exactitud $(Q)$.

III. Regularizar las líneas $Q$ y $X$. Para ello, se deben eliminar aquellos detalles discordantes producidos por aspectos como tamaño, forma, complejidad, número de segmentos en los elementos lineales, etcétera, que perturbarían el resultado final. Su eliminación se logra mediante un proceso de generalización sobre cada pareja de líneas a partir de valores sobre la exactitud del mapa y de la referencia.

IV. Cálculo de la distancia de Hausdorff dH. Ésta se obtiene mediante el cálculo de $\mathrm{d}_{1}$ como el máximo de la distancia mínima de la poligonal a comprobar a la referencia $(X->Q)$ y d $\mathrm{d}_{2}$ como el máximo de la distancia mínima de la referencia a la poligonal a comprobar $(Q->X)$ (Figura 1). Sobre ellas se define la distancia $d_{H}$ como el máximo de las dos componentes anteriores. 
V. Cálculo de las distancias medias a partir de las distancias mínimas de las poligonales de la cartografía a comprobar a las de la referencia. Esta métrica es añadida al método original en Mozas et al. (2007).

En Mozas y Ariza (2008) se utiliza este método con líneas a las cuales se aplicaron errores controlados (líneas sintéticas), concluyendo que este método ofrece información sobre el error máximo alcanzado, así como de la media de errores entre los vértices de las líneas. Tras la aplicación del método a varias líneas a las que se les añadió diferentes tipos de errores, el autor deduce que este método puede dar información de la existencia de errores puntuales o falta de exactitud en la determinación de los inicios y finales de líneas.

Según Mozas (2007), este método se encuentra implementado en la herramienta de software CPLin. También menciona que la principal ventaja de este método respecto a otros radica en que los cálculos necesarios son menores. Sin embargo, los resultados sólo ofrecen información sobre el porcentaje de acuerdo entre ambas líneas (ratio entre las poligonales regularizadas de la cartografía y las líneas iniciales) y la cuantía del RMSE.

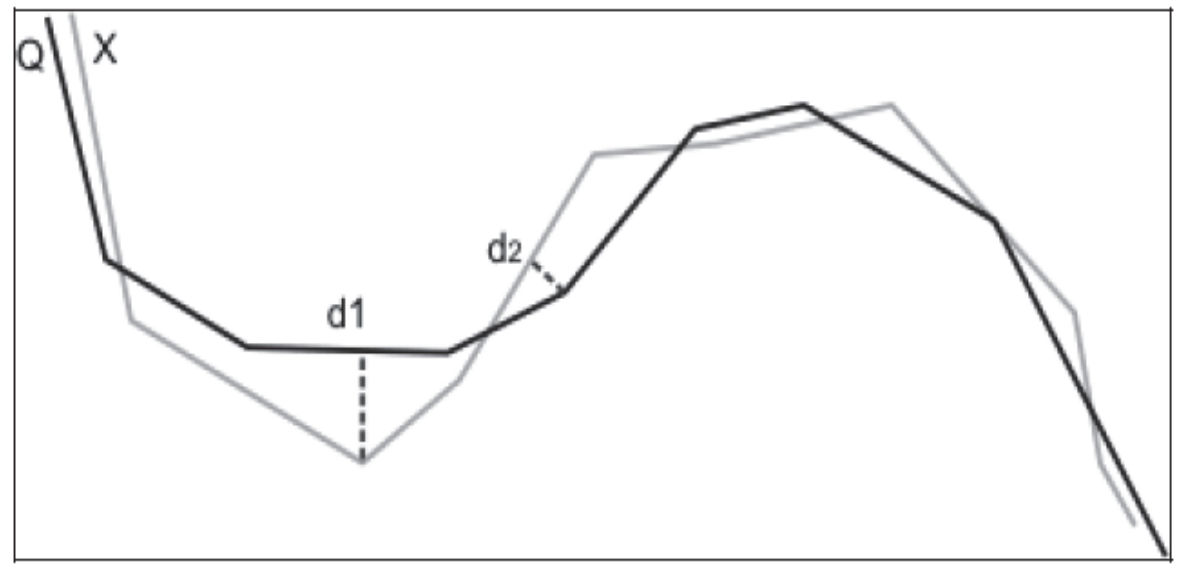

Figura 1. Método de la distancia de Hausdorff. La distancia de Hausdorff es aquella que es la máxima entre la $\mathrm{d}_{1}$ y la $\mathrm{d}_{2}$.

Fuente: Mozas y Ariza (2008).

\section{Método de la Banda Épsilon}

Según Zhang y Goodchild (2002), el concepto de banda épsilon fue planteado por Perkal (1956). Se ha utilizado para describir la inexactitud de la posición de líneas digitalizadas, donde la posición verdadera de una línea se presenta dentro de un cierto desplazamiento (épsilon) de la posición medida. 


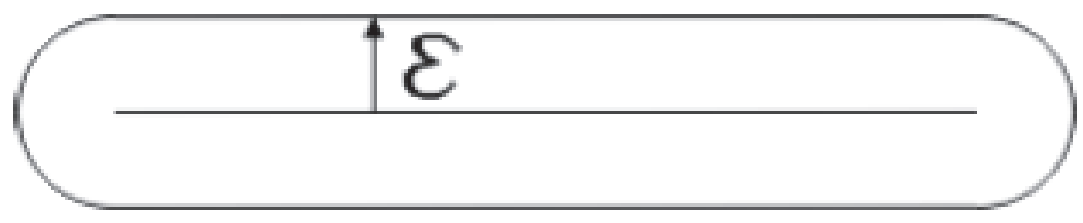

Figura 2. Representación de la banda épsilon sobre una línea recta. Fuente: Mozas (2007).

Según Giordiano y Veregin (1994), la banda épsilon es un tipo de distribución normal centrada sobre la línea o borde de un polígono. Se asume que la posición real de la línea sobre el terreno contenida dentro de épsilon tiene una probabilidad de 1. La anchura de la banda está determinada por el valor de épsilon, que define el grado de incertidumbre relacionada con la posición real de la línea (Figura 2). Cuanto más grande es el valor de épsilon, mayor es la incertidumbre.

Como práctica corriente especificada en este tipo de métodos, la estimación de los valores de épsilon se realiza comparando el dato con fuentes de mayor exactitud. El procedimiento a llevar a cabo es el siguiente:

I. Seleccionar una muestra de líneas en la base de datos $(X)$.

II. Seleccionar las mismas líneas en la otra fuente de mayor exactitud $(Q)$.

III. Para cada línea $X i$ medir el área total de desplazamiento entre la línea del mapa $(X i)$ y la línea de mayor exactitud $(Q i)$. Se debe tener en cuenta que los comienzos y finales de las líneas se unen y que se consideran todas las áreas encerradas por las líneas.

IV. Para cada línea, dividir el área por la longitud de la línea sobre el mapa $(X i)$ para calcular un valor aproximado de épsilon.

V. El valor resultante para un gran número de parejas de líneas forma una distribución estadística de los valores de épsilon. Se pueden calcular diversos parámetros de la distribución, incluso la media o el valor medio cuadrático de épsilon. Como indican Giordano y Veregin (1994), si se asume una distribución centrada en torno a la línea, épsilon define una zona que tiene probabilidad igual a 1 de contener la posición real de la línea sobre el terreno.

En Mozas y Ariza (2008) se emplea este método con líneas a las cuales aplicaron errores controlados (líneas sintéticas), aportando un valor de desplazamiento promedio a partir de la superficie encerrada por las mismas para analizar los resultados. Atkinson y Ariza (2002) subrayan que esta metodología resulta interesante al ser la primera en desarrollarse. Su implementación no supondría gran esfuerzo dentro de un SIG. Sin embargo, no se tienen en cuenta errores a priori del mapa ni 
de la fuente de mayor exactitud, ni se menciona ningún nivel de confianza con el que obtener los resultados.

Mozas (2007) indica que se ha implementado este método en la herramienta software CPLin.

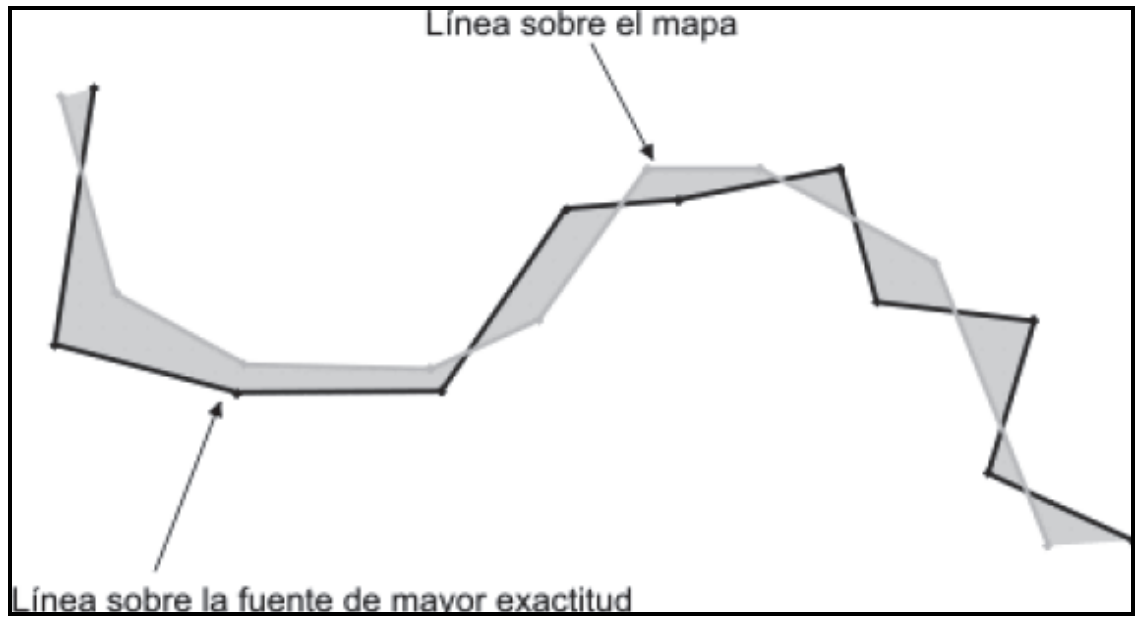

Figura 3. Método de banda épsilon. La distancia épsilon se calcula como el cociente entre el área gris dividido entre la longitud de la línea verde $(X i)$.

Fuente: Mozas y Ariza (2008).

\section{Método de orlados simples}

Este método está basado en los estudios desarrollados por Goodchild y Hunter (1997). El método se fundamenta en la generación de orlados o buffers alrededor de la línea de la fuente de mayor exactitud, contabilizando el porcentaje de longitud de la otra línea del mapa que se encuentra dentro de este orlado.

El procedimiento consiste en:

I. Seleccionar una muestra de líneas en la base de datos $(X)$.

II. Seleccionar estas mismas líneas en la fuente de mayor exactitud $(Q)$.

III. Generar un orlado de anchura $X$ metros sobre las líneas de la fuente de mayor exactitud $(Q)$.

IV. Contabilizar el percentil (\%) de la longitud de las líneas del mapa que se encuentra dentro del orlado.

V. Incrementar la anchura del orlado y repetir el proceso desde el punto 3.

Como indica el autor, a raíz de esto se obtiene una distribución de probabilidades de pertenencia del conjunto líneas del mapa al orlado sobre la fuente de mayor 
exactitud, de forma que se puede discriminar para un percentil que se establezca como umbral la anchura de indeterminación. La representación gráfica de estos resultados genera una curva que aumenta monótonamente a medida que lo hace la anchura del orlado.

Según Atkinson y Ariza (2002), este método resulta bastante robusto y fácil de implementar en SIG. Sin embargo, los autores reconocen que uno de sus principales defectos es que no tiene en cuenta a qué lado de la línea de la fuente de mayor exactitud se encuentra la línea evaluada, por lo que no es posible detectar posibles errores sistemáticos.

Al igual que los métodos anteriores, éste también está implementado en CPLin herramienta software mencionada en Mozas (2007).

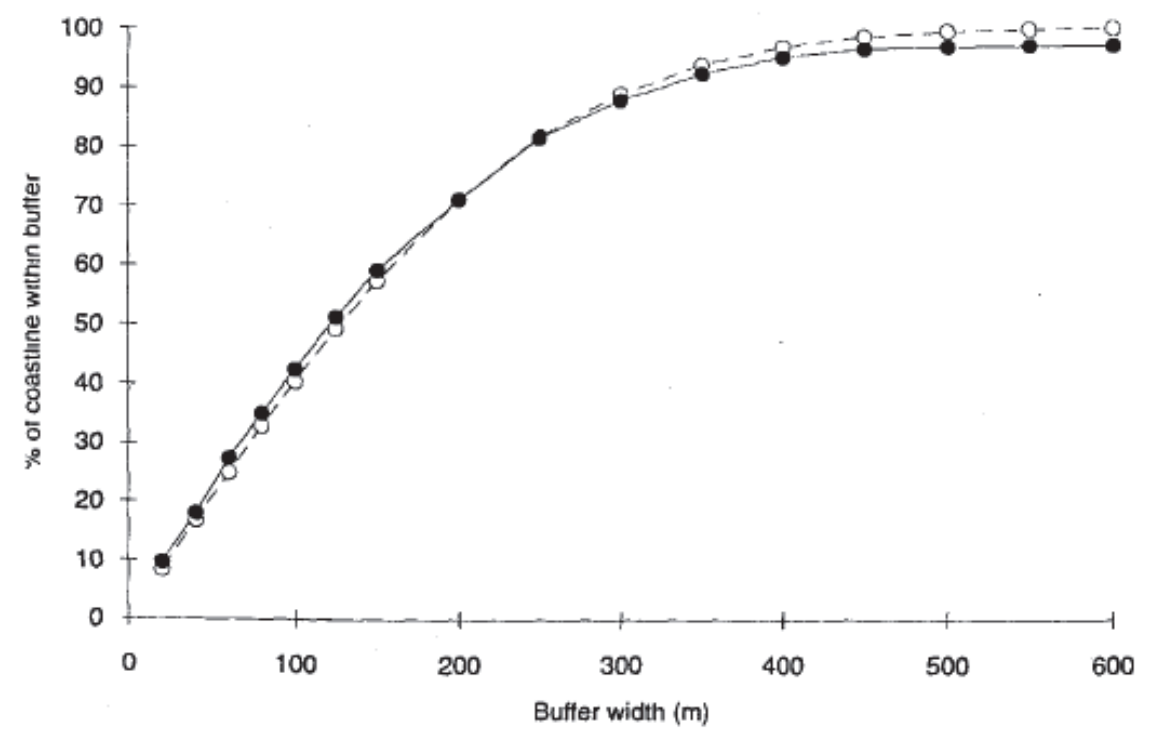

Figura 4. Representación gráfica de los resultados obtenidos por Goodchild y Hunter para una línea en concreto.

Fuente: Goodchild y Hunter (1997).

\section{Método de doble orlado}

El método del doble orlado fue ideado por Tveite y Langaas (1999), aunque ellos lo denominan BOS (Buffer Overlay Statistics Method). Este test puede ser usado para evaluar la anchura de las bandas épsilon, el desplazamiento medio, el nivel de generalización y la compleción. El método se fundamenta en la generación de orlados, aunque es algo más complicado que el método del orlado simple. Esto es debido a 
que el proceso de orlado (buffer) se realiza sobre varias líneas: en la cartografía a analizar $(X)$ y en la fuente de mayor exactitud $(Q)$.

El procedimiento para aplicar el método sigue un proceso iterativo:

I. Seleccionar una muestra de líneas en el mapa $(X)$.

II. Seleccionar estas mismas líneas en la fuente de mayor exactitud $(Q)$.

III. Calcular la longitud de los conjuntos de líneas Long $(X)$ y Long $(Q)$.

IV. Para un orlado de anchura bsi metros se realiza:

i. Orlado de los elementos $X$ y $Q$ de las bases de datos obteniendo los polígonos $X B i$ y $Q B i$ de tamaño 2 bsi.

ii. Proceder a la superposición de los polígonos generando el nuevo conjunto de polígonos: $X B Q B i$.

iii. Calcular las áreas de tipo 2, 3, y 4 y los estadísticos de interés (desplazamiento promedio, oscilación, compleción y sin codificación).

V. Incrementar el tamaño de orlado $(b s i+\Delta)$ y repetir el proceso desde el punto 4 .

VI. Obtener y analizar los resultados, estudiando los gráficos obtenidos.

Gráficamente, las distintas superficies propuestas muestran una curva que disminuye hasta estabilizarse a medida que se aumenta la anchura del orlado (Figura 5).

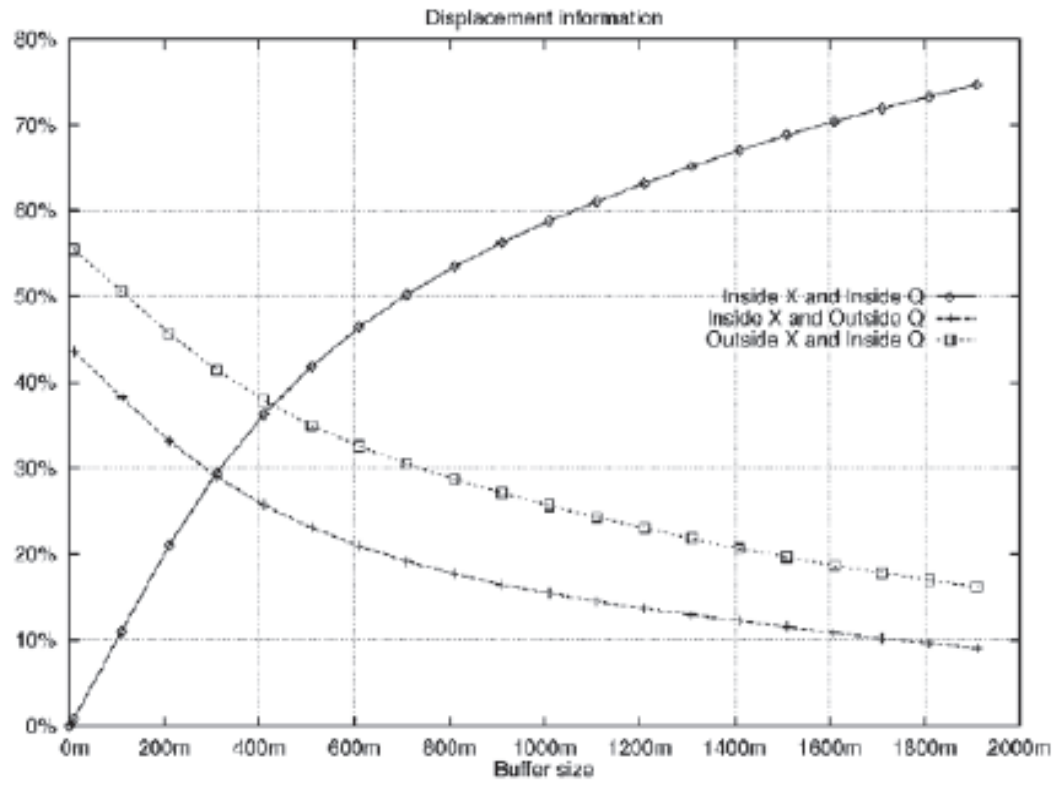

Figura 5. Doble orlado. Comportamiento de las distintas áreas.

Fuente: Tveite y Langaas (1999). 
Tveite y Langaas (1999) presentan algunas medidas evaluadoras de la exactitud posicional a través de este método. Estas medidas son el desplazamiento promedio para cada tamaño de orlado que se usa, el cual sirve para determinar la media de los desplazamientos de una línea $(Q)$ de mayor exactitud sobre una línea $(X)$. También utiliza la medida de oscilaciones aleatorias, que puede ser usada como una indicación de la existencia de errores sistemáticos. Otra medida de la exactitud posicional propuesta por el autor es la de la compleción, que nos indica qué tan completa es la base de datos. Por último, otra de las medidas de exactitud posicional es la que el autor denomina "sin codificar", la cual indica la falta de información de la línea del mapa $(X)$ comparada con la línea de la fuente de mayor exactitud $(Q)$.

Como menciona Mozas (2007), del mismo modo que en el orlado simple, se realiza un proceso iterativo aumentando la anchura del orlado, pero en este caso sobre las líneas de las dos fuentes. Los requerimientos del software son más complejos que en el caso del orlado simple, aunque los resultados ofrecen un análisis más exhaustivo sobre la tipología de los errores, su magnitud y la compleción de la base de datos.

En Mozas y Ariza (2008) se utiliza este método en líneas a las cuales se aplicaron errores controlados (líneas sintéticas), añadiendo como resumen que muestra un porcentaje de solape de los orlados, una medida del desplazamiento promediado y un valor de la oscilación debido a los cruces producidos entre los orlados.

Este método se encuentra implementado en la herramienta software CPLin (Mozas, 2007).

\section{Método de correspondencia de puntos}

Kagawa et al. (1999) describen el denominado por ellos como método de correspondencia de puntos.

El procedimiento que siguen es el siguiente:

I. Generar vértices o puntos virtuales a lo largo de la línea de control $(X)$ con intervalo constante.

II. Desde estos vértices, calcular una aproximación de los vectores de error a la línea de referencia $(Q)$.

III. Determinar o ajustar los vectores de referencia minimizando la suma de los cuadrados de las longitudes.

IV. Durante este procedimiento, establecer condiciones para ordenar los nodos finales de los vectores de error en ambas líneas.

V. Generar un gráfico ordenado de magnitud de errores en cada punto estableciendo el sentido positivo del eje "Y" para los vectores que apuntan a la izquierda siguiendo la dirección de medida. Así, se obtiene una idea de la tendencia de los errores.

VI. Calcular estadísticos sobre los valores de los errores obtenidos. 


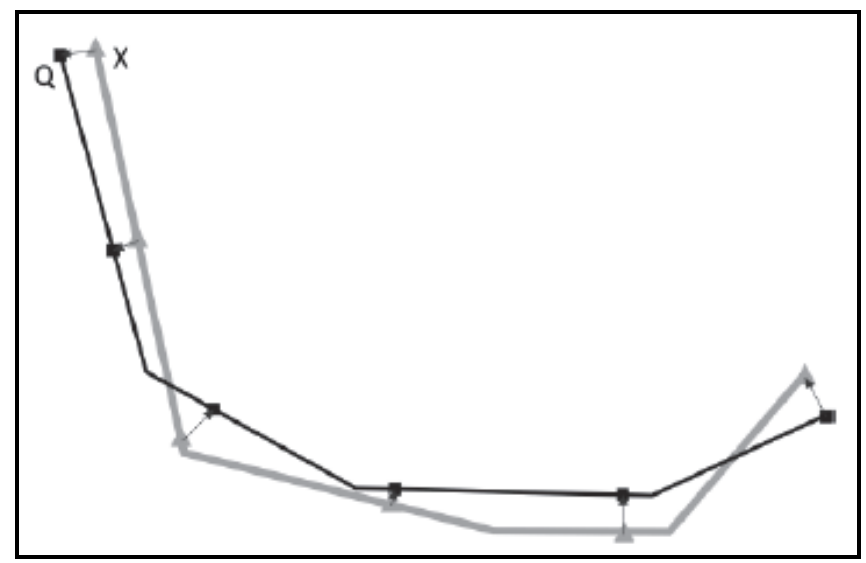

Figura 6. Método de correspondencia de puntos. Se generan puntos a intervalo constante a lo largo de la línea a comprobar y se crean unos vectores de error uniendo con la línea de referencia.

Fuente: Mozas y Ariza (2008).

Kagawa et al. (1999), aplicaron este método en su estudio de carreteras en la ciudad de Sagamihara (Japón). Para una carretera de 706m generan 100 puntos a intervalos constantes y sus vectores a la línea de referencias, minimizando la suma de los cuadrados de las longitudes de los vectores. Los resultados mostraron un error sistemático hacia la izquierda en el sentido de medida y un error medio de $0.68 \mathrm{~m}$.

Según Mozas (2007), como los errores aquí se evalúan en términos de mínimos cuadrados y se asume que los mismos siguen una distribución normal, el cuadrado de los errores se corresponde con la probabilidad de los mismos. Con este método, se pueden estimar errores sistemáticos fácilmente. Además, el mismo autor afirma que es un método interesante aunque exige un tamaño del intervalo constante pequeño, por lo que otro posible problema es que los puntos que se utilizan provienen de la interpolación de puntos medidos (vértices de la línea original, pero que no existen realmente).

\section{Aplicaciones}

Como ya se ha mencionado anteriormente, este tipo de métodos no han sido aplicadas de forma extendida a la hora de realizar controles de calidad, pero sí se han aplicado en casos concretos. Algunos de los más recientes aparecen detallados en los siguientes apartados de este documento.

\section{Catastro}

El método de orlados simples ha sido aplicado a un estudio de catastro por FernándezCastro et al. (2008). 
El estudio ha sido realizado para el ayuntamiento de Guitiriz, provincia de Lugo, España. Además de aplicar el método ya mencionado, también aplica otros de carácter puntual y superficial.

Lo que se hizo fue seleccionar 30 líneas obtenidas a través de trabajos topográficos, siendo éstas las líneas que se tomaron como de mayor exactitud para aplicar el método. Posteriormente, se generaron orlados cada $10 \mathrm{~cm}$ y se contabilizó el porcentaje de longitud de línea cartográfica incluido en cada intervalo, generando una distribución de probabilidades de pertenencia de Q a XBm. De este modo, puede ser, determinado el valor de $\mathrm{m}$ que determina el percentil que se desea establecer como umbral para la anchura de banda de indeterminación correspondiente.

Según señala Fenández-Castro (2008), el autor del método anteriormente descrito no establece un umbral mínimo, sino que deja eso a criterio del usuario, según sus propósitos.

Como resultados, se obtuvo que hizo falta un orlado de $2.2 \mathrm{~m}$ para cubrir la totalidad de las líneas, pero también se puede observar que con un orlado de $0.69 \mathrm{~m}$, 90\% del porcentaje de longitud del conjunto de líneas se encuentra incluido en él.

Estos resultados los pone el autor en contraste con el resultado del test NMAS y el NSSDA. En el caso del primero, la desviación fue de $0.585 \mathrm{~m}$ y del segundo, el RMSE tras eliminar los puntos con mayores desviaciones fue de $0.78 \mathrm{~m}$, valores próximos al tamaño del orlado que incluye $90 \%$ de las longitudes de la líneas.

\section{Líneas de costa}

Goodchild y Hunter (1997) aplicaron el método de orlados simples al cálculo de longitud de líneas de costa.

El estudio fue aplicado a líneas de costa en Australia, obtenidas del Digital Chart of the World (DCW) a escala 1:100,000 comparándolas con las mismas líneas procedentes de un mapa topográfico local a escala 1:25,000. La comparación se hace tras aplicar una transformación de coordenadas para referir ambas a la misma proyección y datum. Los puntos finales de las líneas del mapa de mayor exactitud se enlazaron a los pertenecientes a la cartografía a controlar. Como resumen, se obtuvieron $247 \mathrm{~km}$ en el mapa topográfico que se correspondían con $179 \mathrm{~km}$ del DCW. La diferencia fundamentalmente proviene de la existencia de estuarios de ríos en el mapa topográfico que no aparecen en el DCW. Los resultados mostraron que para $90 \%$ de línea incluido, el tamaño del buffer era de $330 \mathrm{~m}$.

Como añadidura a los métodos mencionados en este artículo, Ramirez y Ali (2003) crearon una serie de medidas de calidad para elementos lineales, las cuales se aplicaron a líneas de costa también. No obstante, estas medidas no cuentan con la complejidad operacional de los métodos mencionados, pero sí pueden ayudar a caracterizar el error en elementos lineales. Estas medidas son: 
I. El factor de sesgo (Bias factor), que evalúa si la línea tiene un sesgo respecto a la de referencia.

II. El factor de distorsión (Distorsion factor), que compara la parametrización estandarizada de dos segmentos de líneas de costa que representan la misma región de la Tierra.

III. Factor de confusión (Fuzziness factor), el cual está relacionado con la definición e identificación de los puntos finales de dos elementos lineales que se quieran comparar.

IV. Factor de generalización (Generalization factor), que compara la longitud total de dos líneas equivalentes, por ejemplo, la líneas de costa generadas por LIDAR y las líneas equivalente obtenidas del modelo digital de elevaciones.

El estudio de Ramirez y Ali (2003) concluyó que estos cuatro métodos se complementan. Los autores detectaron una alta sensibilidad de los modelos a padecer diferentes errores aleatorios y sistemáticos para el caso de las líneas de costa del Digital Government Project. También mencionaron que no estaban seguros de que estos factores evaluaran la mayoría de características de los elementos lineales desde el punto de vista de la exactitud posicional.

\section{Generalización}

El método de distancias de Hausdorff fue aplicado de nuevo por Tsoulos y Skopeliti (1997), pero esta vez para comprobar la eficacia de unos algoritmos de generalización concretos.

El caso de estudio fue aplicado a la línea de costa de la isla griega de Ithaki, digitalizada a escala 1:100,000. Se segmentó toda línea de costa en cinco partes y se clasificaron en tres grupos en función de su sinuosidad. Los algoritmos con los que se simplificaron las líneas fueron los más comunes: Douglas Peucker (DP), Reuman-Witkam (RW) y el de Distancia Euclidania (ED), para varias escalas. Una vez generalizadas todas las líneas con cada uno de los métodos, se contrastaron con las originales de las que partían para comprobar qué error introducía a la línea la aplicación de cada uno de los algoritmos anteriormente mencionados, obteniendo los siguientes resultados tras aplicar el método de distancias de Hausdorff:

- Las líneas más suaves tienen una modificación suave para todos los algoritmos.

- El efecto en la exactitud posicional causado por el algoritmo de Distancia Euclidiana es más moderado comparado con el de Douglas Peucker. El algoritmo de Reuman-Witkam causa mayor desviación horizontal que los otros dos.

- La modificación que genera el aplicar los algoritmos a los tramos sinuosos y muy sinuosos es similar para todos los métodos. 
En resumen, el test encontró mayores distancias de Hausdorff para las líneas más sinuosas que para las líneas menos sinuosas.

\section{Conclusiones}

Existen varios métodos de comprobación de la exactitud posicional a través de elementos lineales, pero lo primero que se debe tener en cuenta a la hora de intentar adoptar uno de ellos a un trabajo personal es que ninguno de ellos está regulado o respaldado por una institución normalizadora. Esto va a impedir que aplicar cualquier método de este tipo tenga una validez a modo de certificación de calidad oficial, y simplemente tendrían utilidad para comprobar la calidad de un documento cartográfico a nivel personal.

A pesar de existir algún método más que los mencionados aquí, todos se basan en los cuatro más extendidos.

Del mismo modo, la aplicación de elementos lineales como soporte para la comprobación de la exactitud posicional en la cartografía está limitada por su complejidad y por la falta de herramientas para aplicarlos si se contrastan con los existentes para elementos puntuales.

Como menciona Ruiz (2009), el rendimiento de la alternativa basada en el control por elementos lineales tiene un costo algo inferior al propio del control puntual, lo que genera que, a pesar de un coste inferior a la hora de obtener mediciones de elementos lineales a través de métodos GNSS, la aplicación de métodos de control basados en elementos lineales puede resultar, según el caso, menos competitiva. Sin embargo, como esto es una opción poco estudiada, los autores consideran que presenta posibilidades de mejora en los rendimientos, tanto en lo que se refiere a trabajo de campo como a técnicas de depuración.

Mozas y Ariza (2008) comenta que los resultados obtenidos para cada una de las metodologías que aplicó a líneas sintéticas muestran comportamientos distintos, con lo que la aplicación de estos métodos puede tener diferentes rendimientos según los diferentes tipos de errores que tengan las líneas a analizar.

\section{Bibliografía}

Ariza Lopez, F.J. (2002). “Calidad en la producción cartográfica”, Universidad de Jaén, Ed. Ra-Ma, Madrid, España, 424 pp.

ASPRS (1990). "Accuracy Standards for Large Scale Maps”, $P E \& R S$, vol. 51, núm. 7, USA, pp. 1068-1070.

Atkinson, A.D.J. y F.J. Ariza (2002). "Nuevo enfoque para el análisis de la calidad posicional en cartografía mediante estudios basados en la geometría lineal", XIV Congreso Internacional de Ingeniería Gráfica, 5-7 de junio, Santander, España, pp. 93-98. 
Fernández Castro, P.; Miranda Barrós, M.; Iniesto Alba, M.J.; Crecente Maseda, R.; Carballo Cruz, P. y F. Reyes (2008). "Evaluación de la calidad posicional de la cartografía catastral española. Un caso de estudio: Guitiriz (Galicia-España)", IX Congreso Internacional de Ingeniería Geomática y Topografía TOP-CART, tomo I, pp. 275-304.

FGDC (1998). "Geospatial Positioning Accuracy Standards", Part 3: National Standard for Spatial Data Acccuracy Subcommitte for Base Cartographic Data, USGS, USA, 25 pp.

Giordano A. y H. Veregin (1994). "Il controllo di qualitá nei sistema informativi territoriali”, Il Cardo, Venecia, Italia, 138 pp.

Goodchild, M. y G. Hunter (1997). "A Simple Positional Accuracy Measure for Linear Features", International Journal of Geographic Information Science, vol. 11, núm. 3, pp. 299-306.

Kagawa, Y.; Sekimoto, Y. y R. Shibaski (1999). "Comparative Study of Positional Accuracy Evaluation of Line Data", $20^{\text {th }}$ Asian Conference on Remote Sensing (ACRS), Hong Kong, China, Poster session 4, 1 p.

Mozas, A.T. y F.J. Ariza López (2008). "Principales métodos de control posicional por elementos lineales. Comparación mediante su aplicación a líneas sintéticas", GeoFocus (Artículos), núm. 8, pp. 187-204.

Mozas, A.T.; Ureña, M.A. y F.J. Ariza (2007). "CPLin: Una herramienta para el control posicional de la cartografía mediante elementos lineales", Revista Mapping, núm. 116, pp. 81-87.

Mozas, A.T. (2007). Control de la calidad posicional en cartografía por elementos lineales, tesis doctoral, Universidad de Jaén, 249 pp.

Perkal, J. (1956). "On Epsilon Length", Bulletin de l'Académie Polonaise des Sciencies, núm. 4, pp. 399-403.

Ramirez, J.R. y T. Ali (2003). "Progress in Metrics Development to Measure Positional Accuracy of Spatial Data", Proceedings of the $21^{\text {st }}$ International Cartographic Conference, Durban, pp. 1763-1772.

Ruiz, J.J.; Mozas, A.T. y M.A. Ureña (2009). "GPS Survey of Road Networks for the Positional Quality Control of Maps", Survey Review, núm. 41, pp. 374-383.

Skidmore, A.K. y B.J. Turner (1992). "Map Accuracy Assessment Using Line Intersect Sampling", Photogrammetric Engineering and Remote Sensing, vol. 58, núm. 10, pp. 1453-1457.

Tsoulos, L. y A. Skopeliti (2000). "Estimating Positional Accuracy of Linear Features", Spatial Information Management Experiences and Visions, FIG Commision 3 Workshop, Athens, pp. 1-5.

Tveite, H. y S. Langaas (1999). "Positional Accuracy Handbook", Governor's Council on Geographic Information. Minnesota, USA, 28 pp. 
USGS (1994). "Standards for a Digital Line Graphs", National Mapping Program. Technical Instructions, Specifications, $65 \mathrm{pp}$.

Zhang, J. y M. Goodchild (2002). "Uncertainty in Geographical Information”, Ed. Taylor \& Francis, London, New York, 288 pp. 